\title{
High-Bandwidth and Sensitive Air Flow Sensing Based on Resonance Properties of CNT-on-Fiber Hairs
}

\author{
Keith Slinker ${ }^{1}$, Corey Kondash ${ }^{1}$, Benjamin T. Dickinson ${ }^{2}$ and Jeffery W. Baur ${ }^{3, *}$ \\ 1 Air Force Research Laboratory, Materials and Manufacturing Directorate, Universal Technology \\ Corporation, Wright-Patterson Air Force Base, Dayton, OH 45433, USA; keith.slinker.ctr@us.af.mil (K.S.); \\ corey.kondash.ctr@us.af.mil (C.K.) \\ 2 Air Force Research Laboratory, Munitions Directorate, Eglin Air Force Base, Valparaiso, FL 32542, USA; \\ benjamin.dickinson.1@us.af.mil \\ 3 Air Force Research Laboratory, Materials and Manufacturing Directorate, Wright-Patterson Air Force Base, \\ Dayton, OH 45433, USA \\ * Correspondence: jeffery.baur@us.af.mil; Tel.: +1-937-260-9415
}

Academic Editor: Jandro L. Abot

Received: 21 December 2016; Accepted: 27 February 2017; Published: 8 March 2017

\begin{abstract}
Artificial hair flow sensors were fabricated using piezoresistive, radially grown carbon nanotube arrays on glass fibers and investigated for their dynamic aerodynamic response as measured within an instrumented plane-wave tube. The sensors were experimentally observed to provide both a large bandwidth of operation below first resonance and a strong resonance response at selected frequencies above first resonance. The frequency of first resonance was easily tunable by adjusting the length of the exposed hair and could be made to vary from a few hundred hertz to over $13 \mathrm{kHz}$. Higher frequency bands were accessible for a given hair length using higher-order resonance modes, up to five of which were observed. All of the responses were understood and modeled using a vibrating Euler-Bernoulli beam analysis.
\end{abstract}

Keywords: CNT; hair flow sensor; vibration resonance frequency; Euler-Bernouli; cantilever; piezoresistive; harmonic oscillator

\section{Introduction}

In addition to a hair sensor's flow sensitivity and response range, the frequency or range of frequencies for which the sensor is useful is of high importance. Sensitivity has been previously discussed and includes both the ability to measure a small amplitude of air perturbation and the magnitude of response per change in air velocity past this threshold [1-4]. The frequency bandwidth of useful operation has been discussed to a smaller extent. It typically includes the in-phase response recorded from quasi-static deflection (DC) to the first natural resonance of the system. For auditory or sonar applications of hair sensors and more exotic frequency-tuned air flow response schemes, the change in air flow at a specific frequency can be isolated by operating an individual hair sensor at its resonance frequency or with a collection of resonance-tailored sensors effectively serving as a bandpass detector [5-7]. In this case, a strong resonant response would be desired at the frequency of interest. Artificial hair sensors which have a strong and frequency-tunable resonance response as well as high sensitivity before the first resonance could function both as a proportional flow sensor below resonance and as a bandpass detector at the selected resonance frequencies.

A number of artificial hair sensor schemes have been previously developed with different hair dimensions, transduction element types, and performance metrics, with the resonance properties only reported for a few. Krijnen et al. suggested the product of the threshold and frequency response bandwidth as a figure of merit for hair sensors [3]. Their 1-mm-long SU8 polymer hairs were 
mounted on a suspended capacitive membrane which rotated at the base under air flow to give a resonance frequency of $500 \mathrm{~Hz}$. The devices were fabricated using microelectromechanical systems (MEMS) processes, and they have been measured in an air flow tube [8]. Chen et al. also use MEMS processes to fabricate a silicon cantilever arm for $600-\mu \mathrm{m}$-long SU8 hairs that resonate at $3.1 \mathrm{kHz}$ [9]. Tamaddoni et al. demonstrated cantilever bending and a $293 \mathrm{~Hz}$ primary resonance in a sensor with a hydrogel-supported lipid bilayer transducer and a 12-mm-long glass fiber [10], and Sarlo et al. recently investigated arrays of similar sensors and the mapping of velocity [11]. Yilmazoglu et al. recently used arrays of carbon nanotubes (CNTs) as both the piezoresistive transduction element and the hair pillars and reported that the sensors maintained functionality even after deflection at $2 \mathrm{kHz}$ [12].

Here we discuss the frequency response of non-MEMS hair flow sensors consisting of a piezoresistive, radial array of CNTs grown on a glass microfiber hair partially within an electroded glass capillary. The length of the glass fiber hair is adjustable in height to a predictable and tunable first resonance frequency as well as a sensitive response before first resonance. Due to the strong resonance, higher-order resonant modes are accessible at even higher frequencies. The fabrication, sensitivity to steady aerodynamics, internal mechanics, and integration of arrays into aerodynamic structures of these CNT-based sensors have been previously discussed $[1,4,13]$. Essentially, the external aerodynamic loading induces a moment inside the capillary which compresses the radially grown CNT array, providing a sigmoidal resistance change (decrease) as a function of flow velocity or deflection.

In now considering the response as a function of dynamic flow (e.g., frequency of deflection), the natural frequencies of a vibrating Euler-Bernoulli beam are known to be

$$
f_{n}=\frac{1}{2 \pi} \frac{\alpha_{n}^{2}}{L_{E}^{2}} \sqrt{\frac{E I}{\rho A}}
$$

where $I, E, \rho, A$, and $L_{E}$ are the moment of inertia, Young's modulus, density, cross-sectional area, and length of the beam, respectively. This assumes the bending stiffness $(E I)$ and mass per length $(\rho \mathrm{A})$ do not greatly vary along the length of the beam. The $\alpha_{n}$ values are determined from the boundary conditions of the beam. Assuming a beam that is free at one end (moment, shear $=0$ ) and fixed at the other (slope $=0$ ), the first four $\alpha_{n}$ values are 1.875, 4.694, 7.854, and 10.996 [14]. While the total length of the hair $\left(L_{T}\right)$ includes the portion of fiber enclosed by the capillary, this model most closely matches these devices if the beam is considered only as the length of the fiber external to the capillary $\left(L_{E}\right)$ and the hair is assumed to be fixed at the opening of the capillary.

Going further, it has been previously shown that these sensors can be more accurately modeled as beams supported by an elastic foundation $[4,15,16]$. In the case of the dynamic response of the sensor, a stiffness constant $\mathrm{k}$ appears in the time-varying Euler beam equation:

$$
E I \frac{\partial^{4} y(x, t)}{\partial x^{4}}+k y(x, t)+\rho A \frac{\partial^{2} y(x, t)}{\partial t^{2}}=0
$$

where $k$ is zero outside the capillary $(x \geq 0)$. This "partial elastic base" condition can be solved by treating the two sections of the beam independently and matching the solutions at the opening of the capillary. The resonance frequencies are found from the relationship

$$
f_{n}=\frac{1}{2 \pi} \sqrt{\left(\frac{\beta_{n}^{2}}{L_{T}^{2}} \sqrt{\left.\frac{E I}{\rho A}\right)^{2}+\frac{k}{\rho A}}\right.}
$$

where the $\beta_{n}$ values are determined from the boundary conditions [15,17]. In this case, for these devices, the hair is modeled as having a free end outside of the capillary $\left(x=L_{E}\right)$ and a fixed end at the bottom of the capillary $\left(x=L_{E}-L_{T}\right)$. In the absence of an elastic base $(k=0)$, the $\beta_{n}$ values are identical to the $\alpha_{n}$ values in Equation (1) and the natural frequencies are the same as those found from the fixed 
base model but with $L_{E}$ replaced by $L_{T}$. Further, for an infinite value of $\mathrm{k}$ in the elastic base model, the two models predict the same resonance frequencies. Using this approach, the frequency response of the experiment response of the fabricated hair sensors can be compared with their predicted responses.

The nanotubes surrounding the glass fiber inside the capillary act as the elastic base, supporting the fiber along the length of hair internal to the capillary. The value of the stiffness constant can be determined by characterizing the bending and deflection of the hair under mechanical loading or by measuring the electrical response under various loading conditions, noting that the electrical response is uniquely a function of the bending moment on the hair [4]. It has been shown that the relationship between the elastic base stiffness and the compressive modulus of the CNTs $(\kappa)$ can be approximated as

$$
k \equiv \frac{\kappa r \pi}{2(R-r)}
$$

where $r$ is in the radius of the glass fiber and $R$ is the inner radius of the capillary.

\section{Results}

Three sensors were investigated in terms of their frequency response using a plane-wave tube. The air flow was dynamically modulated by an audio speaker connected to a 1-m-long, 5-mm-square inner diameter aluminum alloy tube. The speaker was coupled into to the tube at an angle and driven by a power amplifier receiving a sinusoidal input signal from a lock-in amplifier to expose the hairs to air flows at acoustic frequencies. The sound pressure near the sensor was measured with a microphone mounted at the top of the tube at its lengthwise and widthwise center. A proportional-integral-derivative (PID) controller was used to adjust the output of the speaker to maintain a constant signal on the microphone at each frequency. The sensors were mounted at the bottom of the tube directly below the microphone. Propagating plane waves were expected up to a cutoff frequency of about $34 \mathrm{kHz}$ for this tube [18]. The setup is more completely described in the Materials and Methods Section.

The response of Sensor $1\left(L_{E}=4.00 \mathrm{~mm}\right)$ to dynamically modulated air flow is shown in Figure 1a over five periods at two drive frequencies $(100 \mathrm{~Hz}$ and $350 \mathrm{~Hz})$, along with the corresponding Fourier transformed frequency response. In both cases, the root mean square (RMS) amplitude of the supplied modulation was $300 \mathrm{~Pa}$ RMS as measured by the microphone. At $350 \mathrm{~Hz}$, the hair was in resonance with the air flow, as suggested by the large increase in the magnitude of the resistance change and the sinusoidal response to the sinusoidal input. The magnitude of the Fourier transformed response at the driving frequency is plotted in Figure $1 b$ versus the driving frequency. The square root of the resonance frequencies can be seen to vary linearly with the fixed-base $\alpha_{n}$ of Equation (1) (inset of Figure $1 b$ ), indicating a well-behaved elastic response.

To investigate the relationship between the vibrational response of the sensors and their exposed hair length, a second sensor (Sensor 2) was incrementally reduced from an initial $L_{E}$ of $4.73 \mathrm{~mm}$ to a final $L_{E}$ of $0.83 \mathrm{~mm}$. This sensor was one of 25 sensors previously examined in steady flow conditions [4]. The magnitude of the Fourier transformed response is plotted versus the frequency in Figure 2a for each length. Up to five resonance peaks are visible at the initial hair length and progressively shift to higher frequencies as $L_{E}$ is reduced as expected. The peaks corresponding to the primary mode can be fit to the expression for a damped oscillator with a small damping coefficient (e.g., 0.008 for $L_{E}$ of $2.78 \mathrm{~mm}$ ). The resonance frequencies were extracted and are plotted versus the exposed length in Figure $2 b$. 
(a)
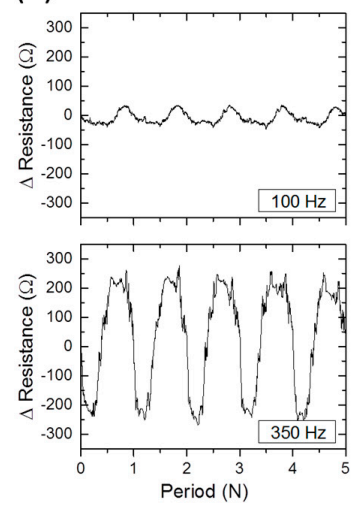
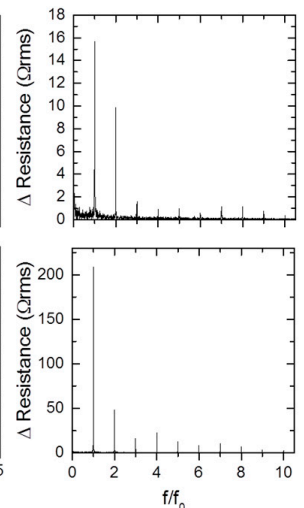

(b)

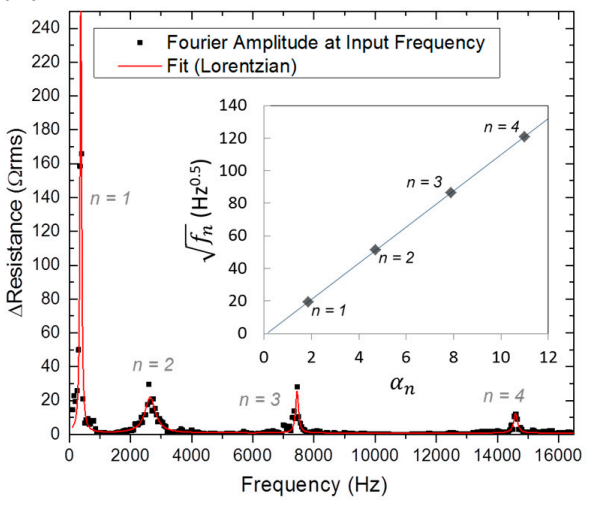

Figure 1. The response of Sensor 1 with $L_{E}=4.00 \mathrm{~mm}$ to dynamically modulated air flow: (a) The change in resistance at $100 \mathrm{~Hz}$ and $350 \mathrm{~Hz}$ over five periods and the corresponding Fourier transformed frequency response scaled by the input frequency $\left(f_{0}\right)$. (b) The amplitude of the Fourier transformed response at the input frequency versus the input frequency. The peaks correspond to the first four resonance modes $(n)$ of the hair sensor. (Inset) The square root of the resonance frequencies $\left(f_{n}\right)$ versus the amplitude factors of the fixed-free Euler-Bernoulli beam with a linear fit.

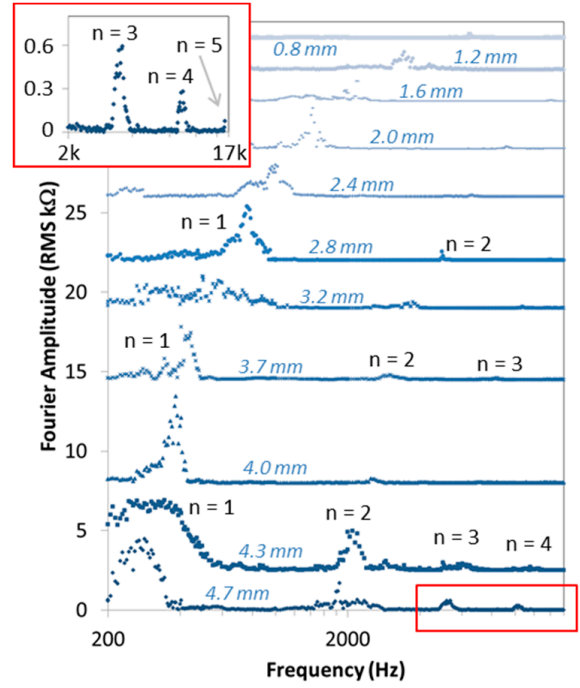

(a)

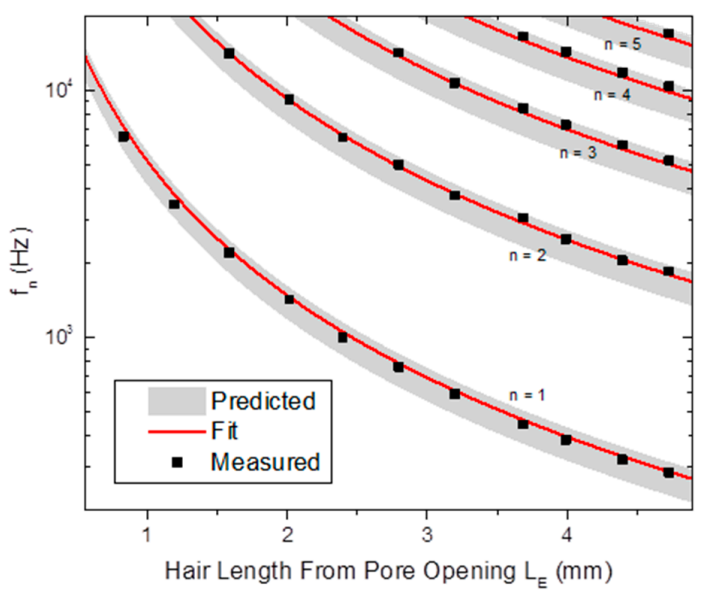

(b)

Figure 2. (a) The amplitude of the Fourier transformed response of Sensor 2 at the input frequency versus the input frequency for 11 hair lengths. Data sets all have minimums of near-zero RMS kW, but are offset in resistance for clarity. $L_{E}$ is indicated for each to the nearest $0.1 \mathrm{~mm}$. The resonance peaks are labeled with their mode number and progressively shift to higher frequencies as the length is decreased. (inset) The high-frequency portion of the response at an $L_{E}$ of $4.7 \mathrm{~mm}$. (b) The resonance frequencies $f_{n}$ versus the exposed length of the hair. The solid red lines correspond to a $\rho A$ of $1.9 \times 10^{-7} \mathrm{~kg} / \mathrm{m}$ and the gray band shows the predicted frequencies for a range of CNT masses.

To assess the minimum detectable threshold of sensors such as these, the amplitude of the response is shown in Figure 3a for Sensor $3\left(L_{E}=4.37 \mathrm{~mm}\right)$ versus the measured sound pressure amplitudes $\left(p_{0}\right)$. The peak air velocity $\left(u_{\text {peak }}\right)$ calculated from the sound pressure

$$
u_{\text {peak }}=\frac{p}{u_{s} \rho_{\text {air }}}
$$


is also indicated, where $\mathrm{p}$ is the pressure amplitude, $u_{s}$ is the speed of sound, and $\rho_{\text {air }}$ is density of air [18]. The three frequencies shown below are the first resonance frequency. The RMS noise level of the setup was seen to be about $2.9 \Omega \cdot \mathrm{rms}$ with no detectable change in resistance above this value for low air velocities. The resistance change increased linearly above a threshold of $70 \mathrm{~mm} \cdot \mathrm{s}^{-1}$ air velocity, which is considered the dynamic detectable threshold for this sensor and measurement setup.

At a much shorter external length $(0.62 \mathrm{~mm})$, the sensitivity was decreased but the first resonance bandwidth was greatly increased. The frequency of the sinusoidal plane wave was swept from $100 \mathrm{~Hz}$ to $16 \mathrm{kHz}$ at constant sound pressure amplitude of $300 \mathrm{~Pa}$. The RMS change in the resistance of the sensor was seen to be approximately constant with the increasing frequency until the frequency approached the first resonance of the sensor at $13.9 \mathrm{kHz}$ and the response of the sensor reached a peak (Figure $3 b$ ). A small peak was also seen around half this frequency due to the increased contribution of the higher modes of the input in the plane wave tube at integer multiples of the applied frequency. The response of 6 to $9 \Omega \cdot \mathrm{rms}$ below resonance is above the noise limit, making this a viable sensor for ultra-large-bandwidth applications even though the cross-section is greatly reduced.

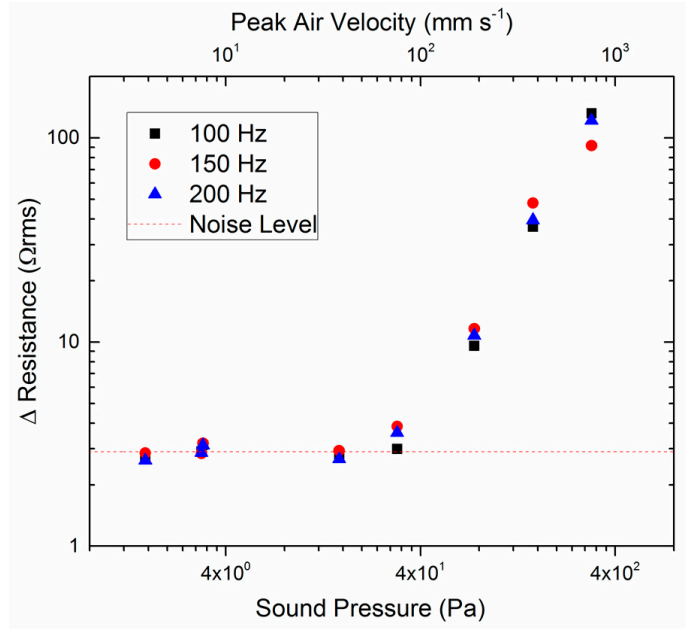

(a)

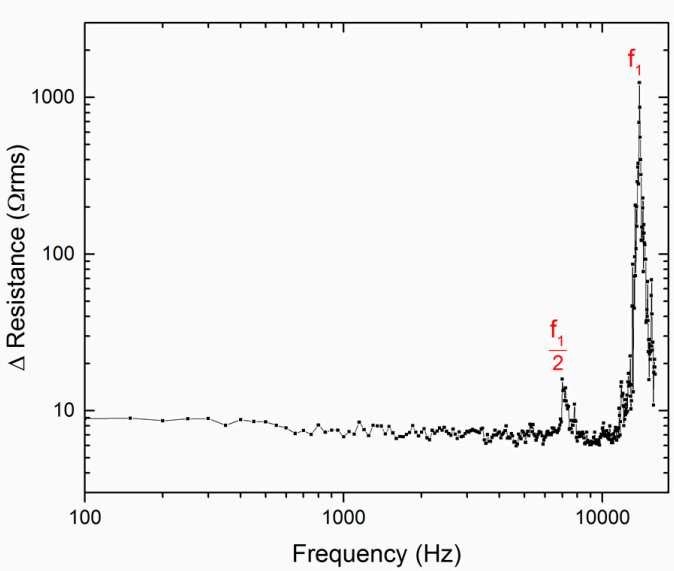

(b)

Figure 3. (a) The RMS change in resistance of Sensor $3\left(L_{E}=4.37 \mathrm{~mm}\right)$ as function of sound pressure amplitude from or air velocity with instrument noise floor of $2.9 \Omega \cdot \mathrm{rms}$. The response increases linearly for air velocities above $70 \mathrm{~mm} \cdot \mathrm{s}^{-1}$. (b) After reducing the $\mathrm{L}_{\mathrm{E}}$ of Sensor 3 to $0.62 \mathrm{~mm}$, the first resonance $f_{1}$ is increased to $13.9 \mathrm{kHz}$ and the response is relatively flat $(3 \Omega \cdot \mathrm{rms}$ minimum) before that resonance. There is an additional peak at half the resonance frequency, but this is believed to be due to the increased presence of higher modes within the plane wave tube.

\section{Discussion}

It can be seen from Equation (1) or Equation (3) that the resonance frequency will vary inversely with the square root of the mass per length of the beam $(\rho A)$. For the $\mathrm{S} 2$ glass microfiber used in these experiments, $\rho A$ is $1.6 \times 10^{-7} \mathrm{~kg} / \mathrm{m}$ as calculated from the reported density. It is expected to be increased by the additional mass of the nanotubes. To determine the upper bound on $\rho \mathrm{A}$, the typical density of the CNT array $\left(\rho_{\mathrm{CNT}}=0.5 \mathrm{~g} \cdot \mathrm{cm}^{-3}\right)$ was estimated from mass and volume change measurements of CNT growth by the same method on large planar substrates. This density is somewhat larger than many reported values for CNT arrays since iron is continually fed to the system during synthesis, resulting in nanoparticles of iron within the volume of the CNT array [19]. The areal density is assumed to be preserved for the glass fiber such that the additional mass per length on a cylindrical fiber is given by $2 \pi \cdot r_{\text {fiber }} \cdot \mathrm{L}_{\mathrm{CNT}} \cdot \rho_{\mathrm{CNT}}$. The average length of the CNTs $\left(\mathrm{L}_{\mathrm{CNT}}\right)$ was measured to be $12 \mu \mathrm{m}$, so the predicted maximum $\rho A$ is $2.7 \times 10^{-7} \mathrm{~kg} / \mathrm{m}$. 
The resonance frequencies predicted by the partial elastic base model in Equation (3) are compared to the measured resonance frequencies in Figure $2 b$. The predicted resonance frequencies are within the bounded range of CNT densities expected, from negligible compared to the density of the glass fiber up to the maximum value estimated above. The measured resonance frequencies are found to fall within this band. The solid red line represents the best fit of the elastic base model to the measured resonance frequencies across all the modes and hair lengths. The mass per length is the only fit parameter and the best fit corresponds to a value of $1.9 \times 10^{-7} \mathrm{~kg} / \mathrm{m}$.

The first resonance bandwidth at any given length, e.g., $5.2 \mathrm{kHz}$ at $L_{E}=1 \mathrm{~mm}$, is large compared to other hair sensors. This is most obviously due to the large bending stiffness of the hair coupled with the low mass per density (e.g., the ratio of EI to $\rho A$ ). Similarly, for hair sensors that rotate at the base, the resonance frequency is proportional to the square root of the ratio of the torsional stiffness of the hinge at the base of the hair to the mass per length of the hair $[3,20]$. The hair will move in rotation rather than bending as long as this torsional stiffness is small relative to the bending stiffness of the hair. In both the torsional and bending cases, the bandwidth can be increased by choosing hair materials with large specific stiffness.

Using the elastic base model, the predicted role of the nanotube stiffness in the resonance frequencies of the sensors is shown in Figure 4 assuming the other properties of the sensor are identical to Sensor 3 and with $L_{E}=4.37 \mathrm{~mm}$. Reported values for the measured stiffness of nanotube arrays vary by as much as three orders of magnitude, most likely due to differences in synthesis conditions and substrate characteristics [21-23]. As the stiffness is changed in this system, there is little predicted change to the useful frequency bandwidth before first resonance even though the higher-order modes show an obvious decrease in frequency with decreasing nanotube stiffness.

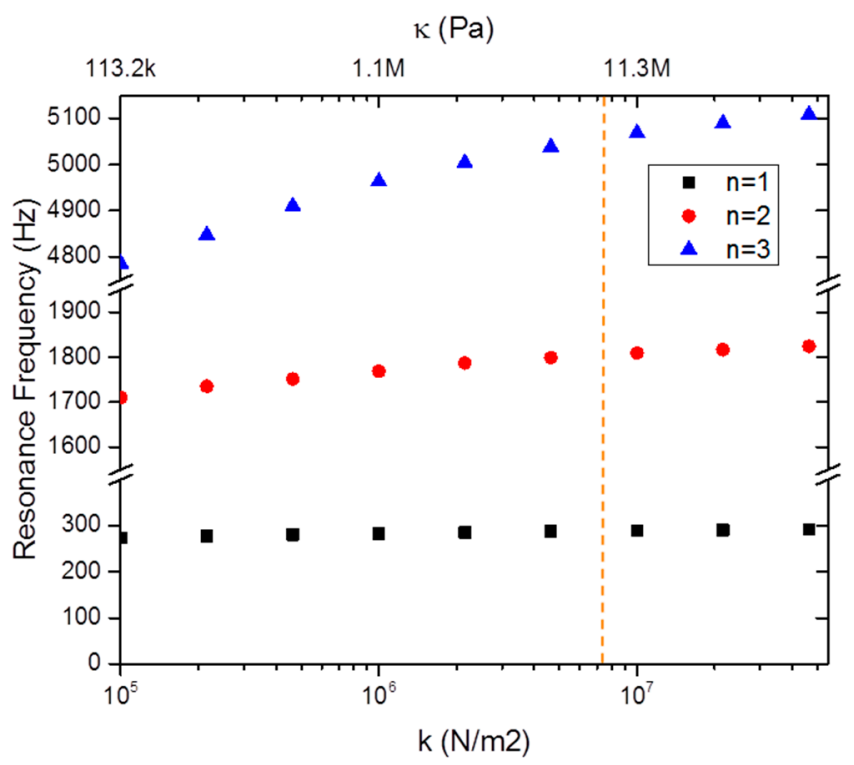

Figure 4. The resonance frequencies predicted for the sensors for various nanotube stiffness values $(\kappa)$ or values of the stiffness-per-length constant in the elastic base model $(k)$. The other properties of the modeled sensors are identical to Sensor 3 with $L_{E}=4.37 \mathrm{~mm}$. The dashed line represents the measured nanotube stiffness of Sensor 3.

Whereas the bandwidth shows little dependence on $k$, the sensitivity and possibly the detectable threshold should be enhanced by decreasing the nanotube array stiffness. Indeed, a quasi-static analysis by Slinker et al. indicates the sensitivity decreases as $k^{1 / 2}$ [4]. Conversely, the quasi-static deflection range over which a change in resistance is significant (i.e., span of the middle section of the sigmoidal response) and the percent resistance change per deflection (the slope of the middle of the sigmoidal response) have opposite dependence on the length and fiber bending stiffness, with the 
former going as $L^{-2}$ and $(E I)^{1 / 2}$, while the latter goes as $L$ and $(E I)^{-1 / 2}$. Control of the stiffness of the support material and alternative means for signal generation (piezoelectric, etc.) may provide opportunities in the future to optimize the sensitivity of these devices without impacting the observed large bandwidth.

\section{Materials and Methods}

\subsection{Sensor Fabrication}

The hairs are glass microfibers (AGY $933 \mathrm{~S}-2$ ) with a radius of $4.5 \mu \mathrm{m}$, a reported density of $2580 \mathrm{~kg} / \mathrm{m}^{3}$, and a reported bending stiffness of $28 \mathrm{N \mu m}^{2}$. The microfibers are uniformly coated with a layer of alumina approximately 10-nm-thick by atomic layer deposition. The pore for the hair is a glass microcapillary with inner radius of $12.5 \mu \mathrm{m}$ which is cut and polished to a final length of $1.2 \mathrm{~mm}$. Gold electrodes are deposited on each end by sputtering at an angle such that the gold coats the inside walls of the capillaries to a depth approximately equal to twice the diameter. The glass microfibers are cut to initial length and inserted into the microcapillaries.

The entire assemblies are subjected to CNT synthesis in a tube furnace at $750{ }^{\circ} \mathrm{C}$ by continuous ferrocene/xylene liquid injection in an argon/hydrogen atmosphere. The CNTs grow preferentially on the alumina-coated glass fiber and radially from the fiber surface as a low density array of wavy, vertically aligned, entangled CNTs. As it grows, the CNT array self-positions the glass fiber near the radial center of the capillary. After CNT synthesis, the hair is fixed to the bottom capillary face with epoxy to prevent axial movement. The completed sensor is then inserted into an electrode polycarbonate disc for flush mounting within the plane-wave tube. Figure 5 shows an example sensor imaged near the opening of the capillary, with some of the nanotubes removed from the glass fiber by focused ion beam. Additional information can be found elsewhere [1,4].

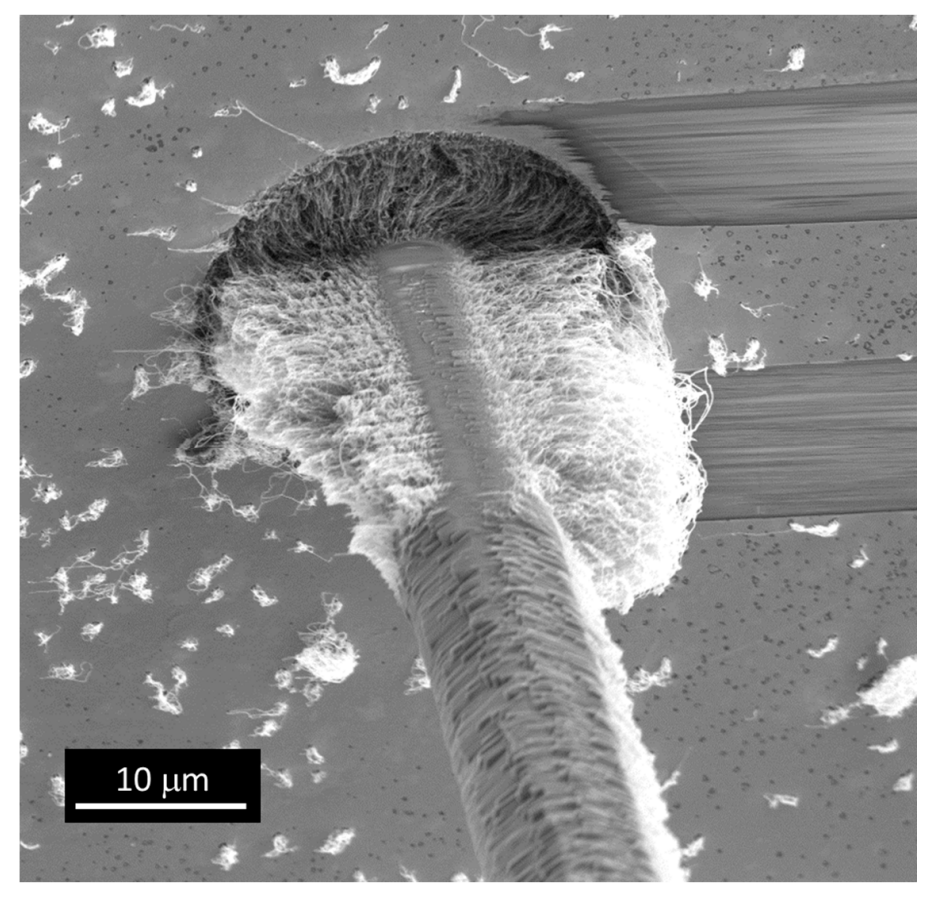

Figure 5. SEM image of a CNT and glass microfiber artificial hair sensor in a metal-coated glass microcapillary pore. CNTs more than about $100 \mu \mathrm{m}$ from the capillary opening were completely removed radially by focused ion beam from the glass fiber for this image, while only the top and bottom CNTs were removed closer to the opening. The radial nanotube array is visible inside the capillary opening. Under normal operation it extends the length of the glass fiber inside and outside the capillary. 


\subsection{Hybrid Plane-Wave Tube Measurements}

A 1-m-long, 5-mm-square inner diameter aluminum alloy plane-wave tube connected on both ends to $8 \mathrm{~m}$ of copper pipe was used to expose the hairs to air flows at acoustic frequencies. A speaker driver (JBL 2446J, 16 ohms) was coupled into one end of the tube. The speaker is driven by a QSC GX3 power amplifier receiving a sinusoidal input signal from Stanford Research Systems SR 830 DSP Lock-In Amplifier. The signal measured by a Brüel \& Kjær 4138 microphone mounted at the top of the tube at its lengthwise and widthwise center was fed to a 2670 preamplifier and recorded by the SR 830. A PID controller was used to adjust the output of the SR 830 to maintain a constant signal on the microphone. The sensors were mounted at the bottom of the tube directly below the microphone. The signals from the microphone and hair sensor were recorded by National Instruments Signal Acquisition Modules NI PXI-4472 (for the microphone) and NI PXI-6224 (for the AHS). The complete setup is shown in Figure 6.

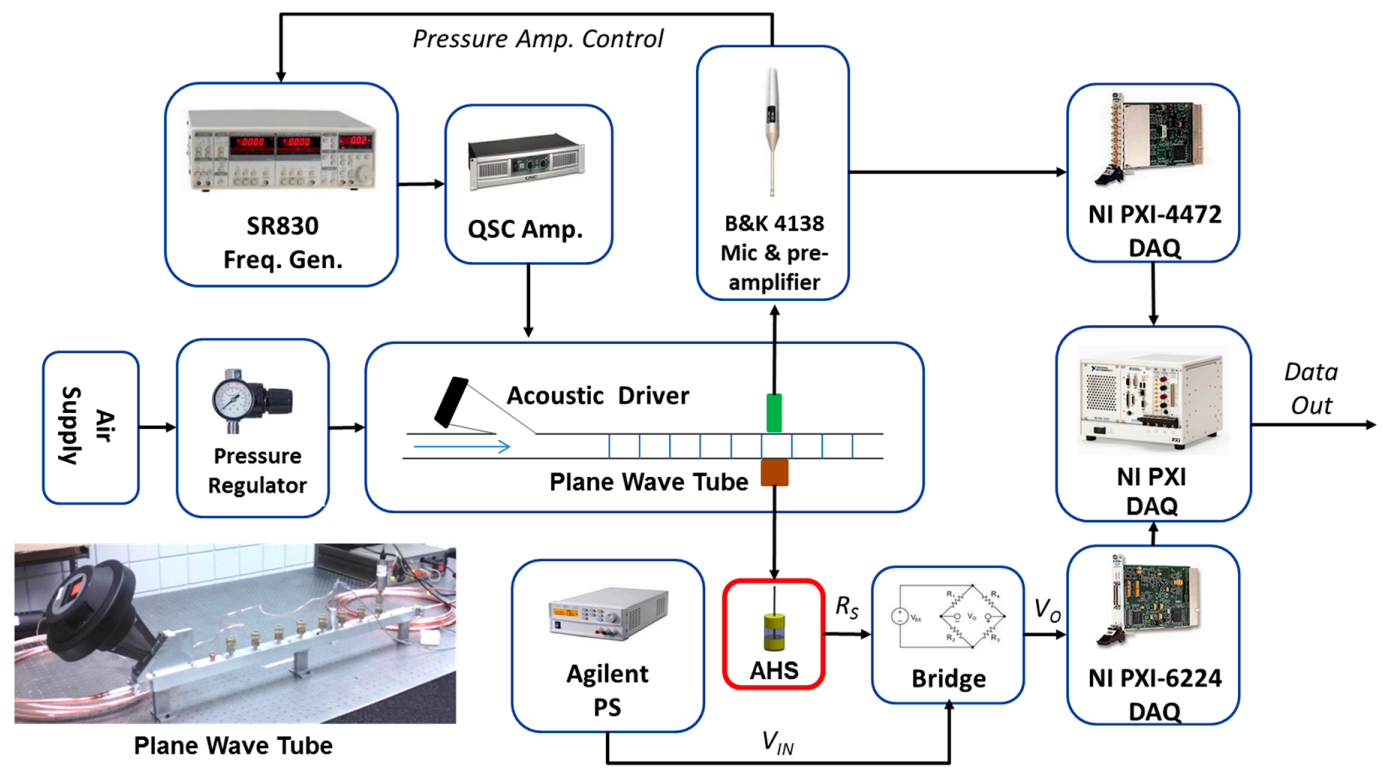

Figure 6. Hybrid plane wave tube for steady and dynamic air flow tests.

Acknowledgments: The authors gratefully acknowledge the Air Force Office of Scientific Research (AFOSR), Byung-Lip (Les) Lee, Program Manager, for financial support of this research, Gregory Reich for discussions and collaborations regarding sensor performance for applications, Benjamin Severin and Peter Schumann for help setting up and running the equipment, and Matthew Maschmann for assistance with the experiments and the focused ion beam processing and imaging.

Author Contributions: Keith Slinker, Benjamin T. Dickinson and Jeffery W. Baur conceived and designed the experiments; Keith Slinker and Corey Kondash performed the experiments; Keith Slinker analyzed the data; Keith Slinker and Jeffery W. Baur wrote the paper.

Conflicts of Interest: The authors declare no conflict of interest.

\section{References}

1. Maschmann, M.R.; Ehlert, G.J.; Dickinson, B.T.; Phillips, D.M.; Ray, C.W.; Reich, G.W.; Baur, J.W. Bioinspired carbon nanotube fuzzy fiber hair sensor for air-flow detection. Adv. Mater. 2014, 26, 3230-3234. [CrossRef] [PubMed]

2. Chen, J.; Fan, Z.; Zou, J.; Engel, J.; Liu, C. Two-dimensional micromachined flow sensor array for fluid mechanics studies. J. Aerosp. Eng. 2003, 16, 85-97. [CrossRef]

3. Krijnen, G.; Floris, A.; Dijkstra, M.; Lammerink, T.; Wiegerink, R. Biomimetic Micromechanical Adaptive Flow-Sensor Arrays. In Proceedings of the SPIE Bioengineered and Bioinspired Systems III, Maspalomas, Gran Canaria, Spain, 2-4 May 2007. 
4. Slinker, K.A.; Kondash, C.; Dickinson, B.T.; Baur, J.W. Cnt-based artificial hair sensors for predictable boundary layer air flow sensing. Adv. Mater. Technol. 2016. [CrossRef]

5. Casas, J.; Dangles, O. Physical ecology of fluid flow sensing in arthropods. Annu. Rev. Entomol. 2010, 55, 505-520. [CrossRef] [PubMed]

6. Junliang, T.; Xiong, Y. Hair flow sensors: From bio-inspiration to bio-mimicking-A review. Smart Mater. Struct. 2012, 21, 11.

7. Magal, C.; Dangles, O.; Caparroy, P.; Casas, J. Hair canopy of cricket sensory system tuned to predator signals. J. Theor. Biol. 2006, 241, 459-466. [CrossRef] [PubMed]

8. Droogendijk, H.; Dagamseh, A.M.K.; Sanders, R.G.P.; Yntema, D.R.; Krijnen, G.J.M. Characterization of bio-inspired hair flow sensors for oscillatory airflows: Techniques to measure the response for both flow and pressure. Meas. Sci. Technol. 2014, 25, 095005. [CrossRef]

9. Chen, N.; Tucker, C.; Engel, J.M.; Yingchen, Y.; Saunvit, P.; Chang, L. Design and characterization of artificial haircell sensor for flow sensing with ultrahigh velocity and angular sensitivity. J. Microelectromech. Syst. 2007, 16, 999-1014. [CrossRef]

10. Nima, T.; Stephen, A.S. Toward cell-inspired materials that feel: Measurements and modeling of mechanotransduction in droplet-based, multi-membrane arrays. Bioinspir. Biomim. 2016, 11, 036008.

11. Sarlo, R.; Najem, J.S.; Leo, D.J. Flow field sensing with bio-inspired artificial hair cell arrays. Sens. Actuators B Chem. 2016, 236, 805-814. [CrossRef]

12. Yilmazoglu, O.; Yadav, S.; Cicek, D.; Schneider, J.J. A nano-microstructured artificial-hair-cell-type sensor based on topologically graded 3d carbon nanotube bundles. Nanotechnology 2016, 27, 365502. [CrossRef] [PubMed]

13. Magar, K.T.; Reich, G.W.; Kondash, C.; Slinker, K.; Pankonien, A.M.; Baur, J.W.; Smyers, B. Aerodynamic parameters from distributed heterogeneous cnt hair sensors with a feedforward neural network. Bioinspir. Biomim. 2016, 11, 066006. [CrossRef] [PubMed]

14. Rao, S.S. Vibration of Continuous Systems; Wiley: Hoboken, NJ, USA, 2007.

15. Slinker, K.; Kondash, C.; Maschmann, M.; Severin, B.; Reich, G.; Dickinson, B.T.; Baur, J. Artificial hair sensors from structural microfibers and cnt arrays for sensing air flow or mechanical shear. In Proceedings of the 20th International Conference on Composite Materials, Copenhagen, Danmark, 19-24 July 2015.

16. Phillips, D.M.; Slinker, K.A.; Ray, C.W.; Hagen, B.J.; Baur, J.W.; Dickinson, B.T.; Reich, G.W. Artificial hair sensors: Electro-mechanical characterization. In Proceedings of the ASME 2014 Conference on Smart Materials, Adaptive Structures and Intelligent Systems, Newport, RI, USA, 8-10 September 2014; p. V002T006A016.

17. Eisenberger, M.; Yankelevsky, D.Z.; Adin, M.A. Vibrations of beams fully or partially supported on elastic foundations. Earthq. Eng. Struct. Dyn. 1985, 13, 651-660. [CrossRef]

18. Chandrasekaran, V.; Cain, A.; Nishida, T.; Cattafesta, L.N.; Sheplak, M. Dynamic calibration technique for thermal shear-stress sensors with mean flow. Exp. Fluids 2005, 39, 56-65. [CrossRef]

19. Futaba, D.N.; Hata, K.; Namai, T.; Yamada, T.; Mizuno, K.; Hayamizu, Y.; Yumura, M.; Iijima, S. 84\% catalyst activity of water-assisted growth of single walled carbon nanotube forest characterization by a statistical and macroscopic approach. J. Phys. Chem. B 2006, 110, 8035-8038. [CrossRef] [PubMed]

20. Shimozawa, T.; Kumagai, T.; Baba, Y. Structural scaling and functional design of the cercal wind-receptor hairs of cricket. J. Comp. Physiol. A 1998, 183, 171-186. [CrossRef]

21. Maschmann, M.R.; Dickinson, B.; Ehlert, G.J.; Baur, J.W. Force sensitive carbon nanotube arrays for biologically inspired airflow sensing. Smart Mater. Struct. 2012, 21, 094024. [CrossRef]

22. Gao, Y.; Kodama, T.; Won, Y.; Dogbe, S.; Pan, L.; Goodson, K.E. Impact of nanotube density and alignment on the elastic modulus near the top and base surfaces of aligned multi-walled carbon nanotube films. Carbon 2012, 50, 3789-3798. [CrossRef]

23. Maschmann, M.R.; Zhang, Q.; Du, F.; Dai, L.; Baur, J. Length dependent foam-like mechanical response of axially indented vertically oriented carbon nanotube arrays. Carbon 2011, 49, 386-397. [CrossRef]

(C) 2017 by the authors. Licensee MDPI, Basel, Switzerland. This article is an open access article distributed under the terms and conditions of the Creative Commons Attribution (CC BY) license (http:/ / creativecommons.org/licenses/by/4.0/). 